\title{
Épidémiologie de la bilharziose urinaire et des geohelminthiases chez les jeunes scolaires des zones lacustres de la commune de So-Ava, sud-Bénin
}

\author{
Ibikounlé Moudachirou1, ${ }^{*}$, Satoguina Judith ${ }^{2}$, Fachinan Rufine ${ }^{1}$, Tokplonou Léonidas ${ }^{2}$, Batcho \\ Wilfrid ${ }^{3}$, Kindé-Gazard Dorothée ${ }^{4}$, Mouahid Gabriel ${ }^{5}$, Moné Hélène ${ }^{5}$, Massougbodji Achille ${ }^{4}$, Courtin \\ David6
}

1Département de Zoologie, Faculté des Sciences et Techniques, Université d'Abomey-Calavi, 01BP526 Cotonou, Bénin

'Laboratoire d'Immunologie des Maladies Infectieuses et Allergiques, Département de Biochimie et de Biologie Cellulaire, Faculté des Sciences et Techniques, Université d'Abomey-Calavi, 01BP526 Cotonou, Bénin 3Programme National de Lutte contre les Maladies Transmissibles, Ministère de Santé 01BP882, Cotonou, BENIN 4Laboratoire de Parasitologie-Mycologie, Faculté des Sciences de la Santé, Université d'Abomey-Calavi, 01BP188 Cotonou, Bénin 5Laboratoire Ecologie et Evolution des Interactions (2EI), UMR 5244 CNRS-UPVD, Université de Perpignan, 52 Avenue Paul Alduy, 66860 Perpignan Cedex, France

6UMR 216-IRD : Mère et enfant face aux infections tropicales, 08 BP 841, Cotonou, Bénin. "Correspondant: Dr Moudachirou IBIKOUNLE, Tél. +229 9764 5885, Fax: +229 2130 0028, Adresse E-mail: mibikounle2001@yahoo.fr

Original submitted in on 23rd July 2013 Published online at www.m.elewa.org on 31 $1^{\text {st }}$ October 2013.

https://dx.doi.org/10.4314/iab.v70i1.98805

\section{RESUME :}

Objectif : Dans les zones intertropicales, les infestations par Schistosoma haematobium et autres espèces d'helminthes sont très répandues et le Bénin ne fait pas exception. Cette étude a pour objectif d'évaluer le statut épidémiologique de la Bilharziose urogénitale et des géohelminthiases chez les enfants d'âge scolaire de la commune de Sô-Ava, Département de l'Atlantique dans le Sud-Bénin.

Méthodologie et Résultats : Le matériel biologique est composé d'urines et de selles provenant de jeunes écoliers âgés de 4 à 8 ans et scolarisés dans les Ecoles Primaires Publiques de Sô-Tchanhoué village, SôTchanhoué Quartier et de Sô-Zounko de la commune de Sô-Ava. Les trois sites de prospection font partie de la commune de Sô-Ava. Six cent quatre échantillons d'urine ont été examinés par la technique de filtration et 363 échantillons de selles examinées par la technique de Kato-Katz. Les résultats d'analyse des urines révèlent les prévalences respectives à $S$. haematobium de $22,22 \%(\mathrm{~N}=153) ; 21,43 \%(\mathrm{~N}=182)$ et $46,47 \%$ ( $N=269)$ dans les trois villages pour une prévalence globale de $32,78 \%(N=604)$ avec autant de garçons que de filles infestés et les enfants de 7 à 8 ans constituent la tranche d'âge la plus touchée. Les résultats d'analyse des selles révèlent trois espèces d'helminthes avec une prévalence globale de $12,12 \%$ $(\mathrm{N}=44)$ de personnes infestées par au moins un des trois parasites Ascaris lumbricoides, Trichuris trichiura et Enterobius vermicularis. Aucun œuf de S. mansoni n'a été observé dans les selles. Les résultats montrent enfin que les charges parasitaires de $S$. haematobium et des géohelminthes étaient plus 
importantes chez les enfants de 7 à 8 ans, en particulier chez les garçons. Les prospections malacologiques ont été réalisées afin d'évaluer la diversité malacologique et déterminer les mollusques impliqués dans la transmission des schistosomes. Trois espèces de mollusques connues comme hôtes intermédiaires de schistosomes ont été collectées Bulinus forskalii, $B$. globosus et $B$. truncatus.

Conclusion : Cette étude a permis d'évaluer le niveau d'endémicité de la bilharziose urogénitale et des géohelminthes au niveau des populations humaines de cette zone lacustre et permet d'envisager les moyens adéquats pour le contrôle de ces parasitoses.

Mots clés : Schistosoma haematobium, Géohelminthes, Sô-Ava, Sud-Bénin, Epidémiologie.

\section{Epidemiology of urogenital schistosomiasis and soil-transmitted helminths among schoolchildren of Lake Nokoue areas of Sô-Ava, south Benin}

\section{Abstract:}

Objective: In the tropics, infections by Schistosoma haematobium and soil-transmitted helminths species are widespread and Republic of Benin is no exception. This study aims to assess the epidemiological status of urogenital schistosomiasis and soil-transmitted helminths in schoolchildren of Sô-Ava town in the Department of Atlantic in Southern Benin.

Methodology and Results: The biological material was composed of urine and stool collected from from Public Primary Schools in Sôchanhoué, Sôtchanhoué Quarter and Sô-Zounko between April to August 2012 . Six hundred and four urine samples were examined by filtration technique and 363 stool samples were examined by Kato-Katz test. Malacological surveys were conducted to assess the diversity of freshwater snails and determine the snail species involved in the transmission of schistosomiasis. The results showed infection rates of $S$. haematobium of $22.22 \%(\mathrm{~N}=153), 21.43 \%(\mathrm{~N}=182)$ and $46.47 \%(\mathrm{~N}=$ $269)$ in the three prospected sites, respectively, with an overall infection rate of $32.78 \%(\mathrm{~N}=604)$ with as many boys as girls infected and children 7 to 8 years of age the most affected. Three species of soiltransmitted helminths were found with an overall prevalence of $12.12 \%(\mathrm{~N}=44)$ of people infected with at least one of the three parasites Ascaris lumbricoides, Trichuris trichiura or Enterobius vermicularis. The results showed that the parasite densities of $S$. haematobium and soil-transmitted helminths were the greatest in children aged 7 to 8 years, especially boys. Three species of freshwater snails known as intermediate hosts for schistosomes were collected: Bulinus forskalii, B. globosus and B. truncatus.

Conclusion: This study was used to assess the level of endemicity of urinary schistosomiasis and soiltransmitted helminths in human populations of the Nokoue Lake area and allows considering the appropriate means of controlling these parasites.

Keywords: Schistosoma haematobium, soil-transmitted helminthes, Sô-Ava, South-Benin, Epidemiology

\section{INTRODUCTION}

La schistosomose, ou Bilharziose est une maladie parasitaire endémique des régions tropicales. Elle est l'une des maladies les plus répandues dans le monde et sévit dans environ 76 pays situés en Afrique, en Amérique du Sud, au Moyen-Orient et au Sud du continent asiatique (WHO 2011). Le nombre de personnes exposées est estimé à 600 millions dont plus de 200 millions sont infestées et près de 280000 personnes décèdent chaque année des complications (Chippaux 2000 ; Chitsulo et al., 2004 ; WHO 2011). Par sa prévalence, la Bilharziose occupe le premier rang des maladies transmises par l'eau et le deuxième rang après le paludisme pour son importance en termes de santé publique dans les régions tropicales et subtropicales (Engels et al., 2002 ; WHO, 2011). Les populations à risque sont : les écoliers, les enfants, les femmes enceintes, les pêcheurs, les agriculteurs qui utilisent la technique de l'irrigation et les éleveurs (Molyneux et al., 2005). Ces populations sont également exposées aux autres helminthes et parasites. La prévalence de ces parasitoses varie selon les régions mais elle est beaucoup plus élevée en zone tropicale. 
Au Bénin, les études épidémiologiques encore parcellaires révèlent la présence de deux espèces de schistosomose : l'espèce $S$. haematobium (forme vésicale) largement distribuée et dont les fréquences d'infestation peuvent varier de $4 \%$ à $100 \%$ et l'espèce S. mansoni (forme intestinale) retrouvée de manière focalisée avec des prévalences pouvant atteindre $60 \%$ (lbikounlé et al., 2009 ; Ibikounlé et al., 2012 ; Ibikounlé et al., 2013). Toutefois, les résultats d'une étude récente

\section{MATERIEL ET METHODES}

Cadre de l'étude : L'étude a été menée dans la Commune lacustre de Sô-Ava, située dans le département de l'Atlantique. La commune de Sô-Ava occupe la Basse-vallée du fleuve Ouémé et de la rivière Sô à qui elle doit son nom (Figure 1). D'une superficie de $218 \mathrm{~km}^{2}$, elle compte 76315 habitants. La densité de la population de Sô-Ava est de 350 habitants par $\mathrm{km}^{2}$ à la date du dernier Recensement Général de la Population et de l'Habitat (RGPH-INSAE, 2012).

Echantillonnage : 604 écoliers ont été sélectionnés de façon aléatoire dans 3 écoles primaires publiques de trois villages Sôtchanhoué (ST : $06^{\circ} 28,337^{\prime} \mathrm{N}$; $02^{\circ} 24,766^{\prime} \quad$ E), Sôtchanhoué-Quartier (STQ : $06^{\circ} 28,337^{\prime} \mathrm{N} ; 02^{\circ} 24,394^{\prime}$ E) et Sô-Zounko (SZ : $06^{\circ} 28,097^{\prime} \mathrm{N} ; 02^{\circ} 24,904^{\prime}$ E) de la commune de SôAva ; toutefois, ils devaient répondre aux critères d'inclusion suivants : (a) être résident permanent dans la zone lacustre, (b) avoir un âge compris entre 4 à 8 ans et (c) avoir fait signé le formulaire de consentement éclairé par leurs parents.

Dépistage de la Bilharziose urogénitale et détermination de la charge parasitaire : Après quelques efforts physiques, toute l'urine de l'écolier est récupérée dans un flacon étiqueté et ramenée au laboratoire dans une glacière réfrigérée. Nous avons utilisé la technique de filtration des œufs. Dix millilitres d'urine homogénéisée sont prélevés à l'aide d'une seringue $10 \mathrm{cc}$ et filtrés à travers d'un filtre. Le filtre est retiré et immédiatement coloré au Lugol puis la lecture des œufs est faite par deux lecteurs à l'objectif x 40 d'un microscope Leica DM 2000.

Dépistage des géohelminthes: La technique utilisée est celle de Kato-Katz (WHO, 1996) qui permet de mentionnent des phénomènes d'introgression génique entre $S$. haematobium et $S$. guineensis (Moné et al., 2012) ; ce qui laisse présager la présence d'une troisième espèce de schistosomes humains au Bénin. La présente étude donne un premier aperçu de la situation épidémiologique des schistosomes et géohelminthiases dans la commune de Sô-Ava, une zone lacustre du sudBénin.

rechercher non seulement les œufs des géohelminthes mais aussi ceux des schistosomes intestinaux comme $S$. mansoni et $S$. guineensis. Elle consiste à filtrer et à étaler $41,7 \mathrm{mg}$ de selles sur une lame porte objet recouverte par une bande de cellophane, trempée 48 heures avant, dans la solution de Kato-Katz (composée de $3 \mathrm{ml}$ de vert de malachite $3 \%$ et $100 \mathrm{ml}$ de glycérol dilué à $50 \%$ avec l'eau distillée). La lecture de la lame est faite deux fois par deux lecteurs à l'objectif $x 40$ d'un microscope DM 2000. La première heure précédant le montage, la lecture est faite pour mettre en évidence les œufs des ankylostomes et vingt quatre heures après pour mettre en évidence les œufs des autres helminthes. Le nombre d'œufs observés pour chaque espèce est multiplié par 24 pour obtenir le nombre d'œufs par gramme de selle, selon les recommandations du fabriquant du Kit.

Prospections malacologiques: Pendant la période de l'étude (de juin à décembre 2012), les prospections ont été menées sur les feuilles flottantes ainsi que dans la vase en vu de rechercher les mollusques gastéropodes connus comme potentiels hôtes intermédiaires de la bilharziose. Les spécimens de mollusques collectés ont été triés et identifiés à l'aide de la clé de détermination de Brown (1994). Chaque catégorie de mollusque est ensuite exposée à la lumière naturelle afin de tester les émissions d'éventuelles cercaires.

Analyses statistiques : Les comparaisons de fréquence sont effectuées à partir du test statistique $Z$ avec le logiciel StatView. Les comparaisons de moyennes sont réalisées à partir du test de Student et de Bartlett à l'aide du logiciel STATA version 8. 


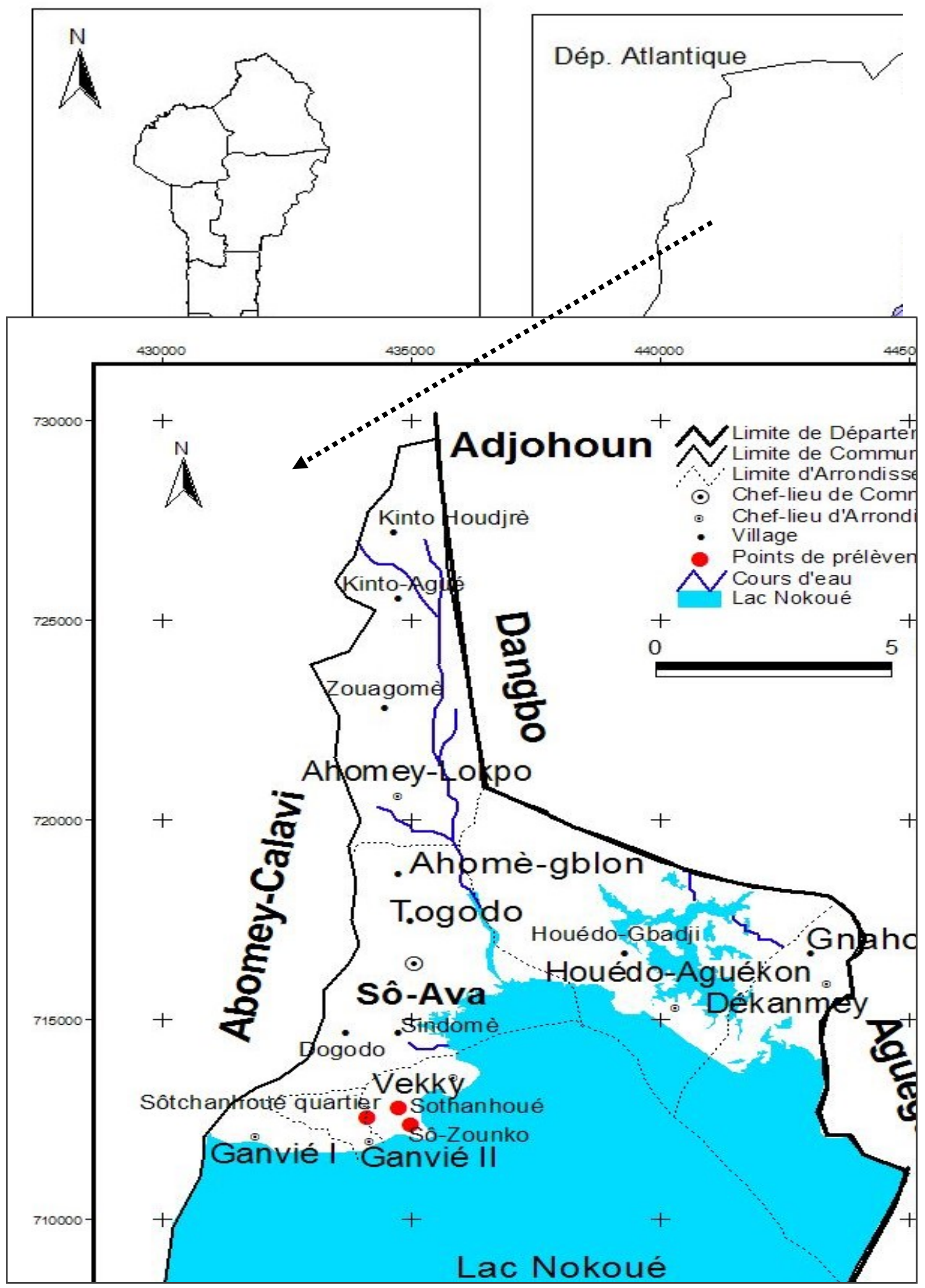

Figure 1 : Situation géographique des écoles enquêtées 


\section{RESULTATS ET DISCUSSION}

Caractéristiques de la population et prévalence de la schistosomose : Les caractéristiques de la population étudiée sont récapitulées dans le tableau 1. Parmi les individus examinés, la présence de $S$. haematobium a été retrouvée dans les 3 villages. Autant de garçons que de filles sont touchés $(p>0,05)$, et les enfants de 7 à 8 ans constituent la tranche d'âge la plus atteinte $(Z=4,193 ; p<0,05)$. Ces résultats confirment l'existence de la bilharziose à $S$. haematobium dans la commune de Sô-Ava, plus précisément dans les villages de Sôtchanhoué, Sôtchanhoué-Quartier et de Sô-Zounko. En effet, sur les 604 sujets examinés, 198 sont infestés soit $32,78 \%$, parmi lesquels $46,47 \%$ sont enregistrés à SZ, $21,43 \%$ à STQ et $22,22 \%$ à ST. Ces résultats sont beaucoup plus bas que ceux obtenus par lbikounlé et al., (2009), qui ont montré une forte prévalence de cette affection, soit $100 \%$ à ST. Cette diminution de taux d'infestation est fortement liée à la sensibilisation de la population et surtout aux campagnes de traitement de masse au Praziquantel organisées dans la commune. Toutefois, la forte proportion d'infestation observée à SZ peut s'expliquer par le fait que la plupart des habitants de cette localité vivent en permanence sur l'eau, quelle que soit la saison. Les résultats de cette étude ont montrés que les enfants de 7-8 ans sont les significativement plus touchés par l'infestation par $S$. haematobium : $38,73 \%$ des enfants de cette tranche d'âge sont infestés contre $21,53 \%$ pour les enfants de 4 à 6 ans $(Z=4,193 ; p<0,05)$, confirmant ainsi les travaux de Etard et al., (1995) au Niger où les enfants âgés de 7-14 ans sont 7 fois plus exposés à l'infestation par $S$. haematobium que les adultes. Cette prédominance de l'infestation à $S$. haematobium chez les jeunes enfants pourrait s'expliquer d'une part par une plus grande mobilité de cette tranche d'âge de la population et d'autre part par une plus grande fréquentation des gîtes à mollusques à travers les activités récréatives. Les plus jeunes, c'est-à-dire ceux âgés de moins de 7 ans, sont toujours sous la surveillance de leurs parents et leur accès à l'eau est souvent contrôlé.

Tableau 1 : Caractéristiques de la population et prévalence de $S$. haematobium

\begin{tabular}{llll}
\hline Caractéristiques & Testés & $\begin{array}{l}\text { Infestés } \\
\mathbf{N}(\%)\end{array}$ & $\begin{array}{l}\text { Non infestés } \\
\mathbf{N}(\%)\end{array}$ \\
\hline Village & & & \\
Sotchanhoué & 153 & $34(22,22)$ & $119(77,78)$ \\
Sotchanhoué Quartier & 182 & $39(21,43)$ & $143(78,57)$ \\
Sô-Zounko & 269 & $125(46,47)$ & $144(53,53)$ \\
\hline Sexe & & & \\
Garçons & 348 & $114(32,76)$ & $234(67,24)$ \\
Filles & 256 & $84(32,81)$ & $172(67,19)$ \\
\hline Age & & & \\
$4-6$ ans & 209 & $45(21,53)$ & $164(78,47)$ \\
$7-8$ ans & 395 & $153(38,73)$ & $242(61,27)$ \\
Total & 604 & $198(32,78)$ & $406(67,22)$ \\
\hline
\end{tabular}

Répartition de la charge parasitaire: La charge parasitaire des enfants infestés par $S$. haematobium est récapitulée dans le Tableau 2. Sur les 172 enfants infestés, 87 ont une forte charge parasitaire. La majorité des écoliers présentant une forte charge parasitaire est rencontrée à SZ, soit 68 contre 13 et 6 respectivement à STQ et ST. On a constaté que la forte charge parasitaire est enregistrée surtout chez les garçons, 55 contre $32(Z=3,336 ; p<0,05)$ et surtout chez les enfants de 7 à 8 ans $(Z=6,064 ; p<0,05)$. Des résultats similaires ont été observés à l'Office du Niger (Coulibaly et al., 2001) qui a trouvé que l'oviurie était significativement plus importante chez les garçons $(p=0,023)$ et au Burkina-Faso (Zongo et al., 2012). 
Tableau 2 : Caractéristiques des enfants infestés en fonction de l'importance de la charge parasitaire

\begin{tabular}{lll}
\hline Caractéristiques & Faible $^{1}$ & Forte $^{2}$ \\
\hline Village & & \\
Sôtchanhoué & $15(71,43)$ & $6(28,57)$ \\
Sôtchanhoué Quartier & $22(62,86)$ & $13(37,14)$ \\
Sô-Zounko & $48(41,38)$ & $68(58,62)$ \\
\hline Sexe & & \\
Garçons & $49(47,12)$ & $55(52,88)$ \\
Filles & $36(52,94)$ & $32(47,06)$ \\
\hline Tranches d'âge & & \\
$4-6$ ans & $24(51,06)$ & $23(48,94)$ \\
$7-8$ ans & $61(48,8)$ & $64(51,2)$ \\
Total & $85(49,42)$ & $87(50,58)$ \\
\hline
\end{tabular}

${ }^{1}$ Faible: $1-49$ œufs/ $10 \mathrm{ml}^{2}$ Forte: $\geq 50$ œufs/ $10 \mathrm{ml}$

Diversité des géohelmintiases : Les différents types d'helminthes retrouvés dans les selles sont récapitulés dans le Tableau 3. Les œufs de trois espèces de géohelminthes ont été rencontrés. II s'agit d'Ascaris lumbricoides, de Trichuris trichiura et d'Enterobius vermicularis. Ascaris lumbricoides est beaucoup plus présent à SZ (13/23) et à STQ. Nous avons aussi constaté que Trichuris trichiura est présent dans les trois quartiers mais avec une prévalence moins importante qu'Ascaris lumbricoides. Enterobius vermicularis n'est présent que sur un ou deux sites à la fois et avec une prévalence négligeable. Trois cas de polyparasitoses répartis comme suit: Enterobius vermicularis et Ascaris lumbricoides; Trichuris trichiura et Ascaris lumbricoides et Enterobius vermicularis et Trichuris trichiura ont été recensés. Ces cas de doubles infestations s'observent plus chez les garçons que chez les filles $(Z=2,744 ; p<0,05)$. Aucun œuf de $S$. mansoni et de $S$. guineensis n'a été observé dans les selles, ce qui pourrait s'expliquer par l'absence ces deux espèces de schistosome dans la zone d'étude.

Tableau 3 : Diversité des géohelminthes retrouvés dans les selles

\begin{tabular}{lllll}
\hline & Village & & & \\
\cline { 2 - 4 } Types de géohelminthes & ST & STQ & SZ & Total \\
\hline Ascaris lumbricoides & 2 & 8 & 13 & 23 \\
Trichuris trichiura & 4 & 4 & 3 & 11 \\
Enterobius vermicularis & 0 & 4 & 1 & 5 \\
\hline
\end{tabular}

Distribution des géohelminthiases dans la population étudiée : Les caractéristiques des enfants infestés par les géohelminthiases sont récapitulées par le tableau 4. La plupart des enfants infestés par les géohelminthes sont scolarisés à SZ et à STQ. Les garçons sont plus touchés et aussi les enfants de 7 à 8 ans, et ceci de façon significative $(Z=2,346 ; p<0,05$; $Z=3,198 ; p<0,05$, respectivement). Les enfants infestés par les géohelminthes sont plus représentés à SZ qu'à STQ et à ST, soit 25 enfants contre 11 et 8 à STQ et ST, respectivement. Environ $12 \%$ des enfants sont infestés. Nous avons constaté que, bien que ces résultats ne soient pas significatifs, les garçons sont plus affectés que les filles, avec un taux de 13,46\% contre $10,32 \%$. Ces résultats sont similaires à ceux de
Menan et al., 1997 en Côte d'Ivoire. Ces résultats montrent également que les enfants de 4-6 ans sont les plus affectés, avec un taux de $13,46 \%$ contre $11,58 \%$ à l'entrée et $20 \%$ contre $9,8 \%$ à la sortie. Ceci peut s'expliquer par le manque d'hygiène sur cette tranche d'âge.

Diversité malacologique : Six espèces de mollusques gastéropodes ont été récoltées dont trois sont connues dans la transmission de $S$. haematobium au Bénin (Ibikounlé et al., 2009 ; Moné et al., 2010): Bulinus forskalii, $B$. globosus et $B$. truncatus, une espèce, Indoplanorbis exustus, connue comme hôte intermédiaire de schistosome du groupe indicum en Asie et deux autres espèces, Physa marmorata et Lymnaea natalentis. 
Tableau 4 : Répartition des géohelminthiases

\begin{tabular}{lll}
\hline Caractéristiques & Infestés N $(\%)$ & Non infestés N $(\%)$ \\
\hline Villages & & \\
Sôtchanhoué & $8(10,53)$ & $68(89,47)$ \\
Sôtchanhoué Quartier & $18(19,57)$ & $74(80,43)$ \\
Sô-Zounko & $18(9,23)$ & $177(90,77)$ \\
\hline Sexe & $28(13,46)$ & $180(86.54)$ \\
Garçons & $16(10,32)$ & $139(89,68)$ \\
Filles & & \\
\hline Tranche d'âge & $14(13,46)$ & $90(86,54)$ \\
$4-6$ ans & $30(11,58)$ & $229(88,42)$ \\
$7-8$ ans & $44(12,12)$ & $319(87,88)$ \\
\hline
\end{tabular}

\section{CONCLUSION}

II ressort de cette étude que seule l'espèce $S$. haematobium est présente dans la zone d'étude avec une prévalence de $32,78 \%$ qui est en nette régression par rapport au taux de 100\% antérieurement signalé en 2009. Trois parasites intestinaux ont été recensés Ascaris lumbricoides, Trichirus trichuira et Enterobius

CONSIDERATION ETHIQUE : Cette étude a été réalisée en collaboration avec le Ministère de la Santé et celui de l'Enseignement Maternel et Primaire du Bénin. Un consentement éclairé a été signé par les

\section{REMERCIEMENTS}

Cette étude est financée par l'IRD à travers le projet JEAI-PALUCO. Les auteurs adressent leurs remerciements à $M$. ODJOU Pierre, Inspecteur Pédagogique de la Commune de Sô-Ava pour avoir autorisé l'étude et M. AÏTCHEDJI Aurel et M. AVLESSI

\section{REFERENCES BIBLIOGRAPHIQUES}

Chippaux JP, 2000. Control of schistosomiasis: realities and futurology. Médecine Tropicale, 60: 54-55.

Chitsulo L, Loverde P, Engels D, 2004. Schistosomiasis. Nat. Rev. Microbiol., 2: 1213.

Coulibaly H, 2001. Evaluations épidémiologiques, étude clinique et enquête sur les connaissances, attitudes et pratiques de la population en matière de bilharziose en milieu périurbain du district de Bamako (djicoroni para). Thèse de médecine, Bamako, 74p.

Engels D, Chitsulo L, Montresor A, Savioli L, 2002. The global epidemiological situation of schistosomiasis and new approaches to control and research. Acta Tropica, 82,139146. vermicularis avec une prévalence globale de $12,12 \%$ Les enfants de 7 à 8 ans sont les plus infestés avec un portage chronique de $S$. haematobium, tandis que les enfants de 4 à 6 ans sont les plus fréquemment infestés par les géohelminthes. Les garçons sont plus exposés au portage des géohelminthes que des filles.

parents ou tuteur de chaque écolier. Un traitement par chimiothérapie au Praziquantel et à l'Albendazole a été réalisé pour tous les cas positifs.

Célestin, respectivement Administrateur et Laborantin du Centre de Santé Saint Joseph qui a assuré la prise en charge des participants.

Etard JF, Audibert M, Dabo A., 1995. Age-acquired resistance and predisposition to reinfection with Schistosoma haematobium after treatment with praziquantel in Mali. Am J Trop Med Hyg, 52: 549-558.

Ibikounlé M, Mouahid G, Mintsa Nguéma R, KindéGazard D, Sakiti NG, Massougbodji A, Moné $\mathrm{H}$, 2012. Life-history traits indicate local adaptation of the schistosome parasite, Schistosoma mansoni, to its snail host, Biomphalaria pfeifferi. Experimental Parasitology, 132:501-507.

Ibikounlé M, Mouahid G, Mintsa Nguéma R, Sakiti NG, Massougbodji A, Moné $H$, 2013. Snail intermediate host/Schistosoma haematobium relationships from three transmission sites in 
Benin (West Africa). Parasitology Research, 112: 227-233.

Ibikounlé M, Mouahid G, Sakiti NG, Massougbodji A, Moné $H, 2009$. Freshwater snail diversity in Benin (West Africa) with a focus on human schistosomiasis. Acta Tropica, 111: 29-34.

Menan EIH, Nebavi NGF, Abjetey TAK, Assavo NN, Kiki-Barro PC, Kone M, 1997. Profil des helminthiases intestinales chez les enfants d'âge scolaire dans la ville d'Abidjan. Bull. Soc. Path. Exot. 90: 51-54.

Molyneux DH, Hotez PJ, Fenwick A, 2005. "Rapid impact intervention": how a policy of integrated control for Africa's neglected tropical diseases could benefit the poor. PLoSMed. 2, e336.

Moné $H$, Ibikounlé $M$, Massougbodji $A$, Mouahid $G$, 2010. Human schistosomiasis in the Economic Community of West African States (ECOWAS): epidemiology and control. Advances in Parasitology 71: 33-91.
Moné $H$, Minguez $S$, Ibikounlé $M$, Allienne JF, Massougbodji A, Mouahid G, 2012. Natural Interactions between $S$. haematobium and $S$. guineensis in the Republic of Benin, The Scientific World Journal, vol. 2012, Article ID 793420, 8 pages.

RGPH / INSAE Résultat provisoire Recensement Général de la Population et de l'Habitat Institut National de la Statistique et de l'Analyse Economique (INSAE), Bénin, $2002 ; 30$.

WHO 1996. Stool examination kit; Kato-katz technique cellophane faecal thick smear.

WHO 2011. Rapport du comité O. M. S. d'experts sur la lutte contre les maladies tropicales négligées.

Zongo D, Kabre BG, Dayeri D, Savadogo B, Poda JN, 2012. Comparative study of schistosomiasis transmission (urinary and intestinal forms) at 10 sites in Burkina Faso (in sub-Saharan Africa). Med Sante Trop, 22: 323-329. 